\title{
Annihilating Ideals of Quadratic Forms over Local and Global Fields
}

\author{
Klaas-Tido Rühl *
}

August 24, 2009

\begin{abstract}
We study annihilating polynomials and annihilating ideals for elements of Witt rings for groups of exponent 2. With the help of these results and certain calculations involving the Clifford invariant we are able to give full sets of generators for the annihilating ideal of both the isometry class and the equivalence class of an arbitrary quadratic form over a local field. By applying the Hasse-Minkowski theorem we can then achieve the same for an arbitrary quadratic form over a global field.
\end{abstract}

\section{Introduction}

The study of annihilating polynomials of quadratic forms originates in the article [Lew87] by David Lewis. Lewis proved that for $n \in \mathbb{N}_{0}$ the polynomial

$$
P_{n}=(X-n)(X-n+2) \cdots(X+n-2)(X+n) \in \mathbb{Z}[X]
$$

annihilates the isometry class of every $n$-dimensional quadratic form over any field $K$ with $\operatorname{char}(K) \neq 2$. Since then a number of classes of quadratic forms have been identified for which there exist annihilating polynomials of lower degree, for example positive quadratic forms ([Lew92]), trace forms ([Con87], [BP97] and [LM00]) and excellent forms ([Rüh08]). Some effort has also been put into determining the ideal consisting of those polynomials which annihilate the whole Witt ring, its fundamental ideal, or its torsion subgroup ([OG97] and [Wan07]). A survey of many of the currently known results about annihilating polynomials of quadratic forms can be found in [Lew01].

In this article we will concentrate on identifying all annihilating polynomials for a given quadratic form $\varphi$ over a local or global field $K$. More specifically we will give a full set of generators for the annihilating ideal of the isometry class as well as the equivalence class of $\varphi$, i.e. the ideal in

\footnotetext{
*The author gratefully acknowledges support from the Swiss National Science Foundation, grant no. 200020109174/1 (project leader: E. Bayer-Fluckiger), and the Sixth Framework Programme of the European Union through the Marie Curie Actions GTEM (Galois Theory and Explicit Methods) Research and Training Network, contract no. MRTN-CT-2006-035495 (partner 6: Lausanne, scientist in charge: E. Bayer-Fluckiger).
} 
$\mathbb{Z}[X]$ consisting of all annihilating polynomials of the isometry class, respectively the equivalence class, of $\varphi$.

We start with some general results about annihilating polynomials (section 3). There exists a natural setting for the study of these polynomials. Instead of only considering elements of the Witt-Grothendieck ring and the Witt ring, we will more generally study annihilating polynomials of elements of Witt rings for groups of exponent 2. Let $R$ be such a ring, and let $x \in R$. We study the general anatomy of annihilating polynomials of $x$ and use these results to describe a method that allows constructing full sets of generators for the annihilating ideal of $x$ such that the generators have certain nice properties.

In section 4 we study annihilating polynomials of quadratic forms over a local field with finite residue field. We use the fact that over such a field $K$ quadratic forms can be completely classified by the dimension, the determinant and the Clifford invariant. With the help of some calculations involving the Clifford invariant we will be able to fully describe the annihilating ideal of both the isometry class and the equivalence class of a quadratic form over $K$. By using the HasseMinkowski theorem we will then achieve the same for the isometry and equivalence classes of quadratic forms over global fields (section 5).

\section{Notation}

Let $\mathbb{N}$ be the set of natural numbers, and let $\mathbb{N}_{0}:=\mathbb{N} \cup\{0\}$.

The reader is expected to be familiar with the algebraic theory of quadratic forms over fields. Introductions to this theory can be found in [Pfi95], [Sch85] or [Lam05].

Throughout this article $K$ will denote a field with $\operatorname{char}(K) \neq 2$. All quadratic forms over $K$ are understood to be non-degenerate. Often we will simply use the notion "form" when we are referring to a quadratic form.

Let $\varphi$ be a quadratic form over $K$. The dimension of its corresponding quadratic space will be denoted by $\operatorname{dim}(\varphi)$. If $\psi$ is another quadratic form over $K$, then we use the notation $\varphi \perp \psi$, respectively $\varphi \otimes \psi$, for the orthogonal sum, respectively tensor product, of $\varphi$ and $\psi$. For $n \in \mathbb{N}_{0}$ we set

$$
n \times \varphi:=\underbrace{\varphi \perp \ldots \perp \varphi}_{n \text {-times }} .
$$

We write $\varphi \cong \psi$ to indicate that $\varphi$ and $\psi$ are isometric. If there exists a form $\chi$ over $K$ such that $\varphi \cong \psi \perp \chi$, then $\psi$ is a subform of $\varphi$.

Let $n=\operatorname{dim}(\varphi)$. Then there exist $a_{1}, \ldots, a_{n} \in K^{*}$ such that $\varphi$ is isometric to a form over $K$ which can be represented by a diagonal matrix with entries $a_{1}, \ldots, a_{n}$. We write $\varphi \cong\left\langle a_{1}, \ldots, a_{n}\right\rangle$. If $\varphi \cong\left\langle 1, b_{1}\right\rangle \otimes \cdots \otimes\left\langle 1, b_{k}\right\rangle$ for some $b_{1}, \ldots, b_{k} \in K^{*}$ and $k \in \mathbb{N}_{0}$, then $\varphi$ is a $k$-fold Pfister form. We use the notation

$$
\left\langle\left\langle b_{1}, \ldots, b_{k}\right\rangle\right\rangle:=\left\langle 1, b_{1}\right\rangle \otimes \cdots \otimes\left\langle 1, b_{k}\right\rangle .
$$

For $b \in K^{*}$ we set $b \varphi$ to be isometric to $b\left\langle a_{1}, \ldots, a_{n}\right\rangle:=\left\langle b a_{1}, \ldots, b a_{n}\right\rangle$. In particular $-\varphi \cong$ $\left\langle-a_{1}, \ldots,-a_{n}\right\rangle$. 
Let $r \in \mathbb{Z}$. If $r \geq 0$ we will often simply write $r$ instead of $r \times\langle 1\rangle$. For $r<0$ we use the notation $r$ in the quadratic form context to mean $-r \times\langle-1\rangle$.

If $L$ is a field extension of $K$, then $\varphi_{L}$ will denote the quadratic form $\varphi$ considered as a form over $L$.

For the form $\varphi$ we denote by $[\varphi]$ its isometry class. The isometry classes over $K$ form a semi-ring $\widehat{W}^{+}(K)$. If we apply the Grothendieck construction to $\widehat{W}^{+}(K)$ we obtain the Witt-Grothendieck ring $\widehat{W}(K)$. Addition and multiplication in $\widehat{W}(K)$ will be denoted by $[\varphi]+[\psi]$, respectively $[\varphi][\psi]$ (or $[\varphi] \cdot[\psi]$ ). We extend the notion of dimension to $\widehat{W}(K)$. If $[\varphi]-[\psi]$ is an arbitrary element of $\widehat{W}(K)$, then we set $\operatorname{dim}([\varphi]-[\psi]):=\operatorname{dim}(\varphi)-\operatorname{dim}(\psi)$.

The quotient $W(K):=\widehat{W}(K) /([\mathbb{H}])$, where $\mathbb{H}:=\langle 1,-1\rangle$ is the hyperbolic plane, is called Witt ring of $K$. If $\varphi$ and $\psi$ have the same images in $W(K)$, then $\varphi$ and $\psi$ are called equivalent and we write $\varphi \sim \psi$. The equivalence class of $\varphi$ is denoted by $\{\varphi\}$. We use the same notation for the addition and multiplication in $W(K)$ as for $\widehat{W}(K)$. The fundamental ideal of $K$ is the ideal $I(K) \subset W(K)$ consisting of all equivalence classes of even-dimensional forms over $K$.

For $a \in K^{*}$ we denote by $\bar{a}:=a\left(K^{*}\right)^{2}$ its class in the square class group $K^{*} /\left(K^{*}\right)^{2}$. If $\varphi \cong$ $\left\langle a_{1}, \ldots, a_{n}\right\rangle$ with $a_{1}, \ldots, a_{n} \in K^{*}$, then it is clear that replacing $a_{i}, i \in\{1, \ldots, n\}$, by any representative of $\overline{a_{i}}$ will yield a form isometric to $\varphi$. Since we are only interested in quadratic forms up to isometry, we allow the notation $\left\langle\overline{a_{1}}, \ldots, \overline{a_{n}}\right\rangle$. The invariant $\operatorname{det}(\varphi):=\overline{a_{1} \ldots a_{n}} \in$ $K^{*} /\left(K^{*}\right)^{2}$ is the determinant of $\varphi$, and we set $d(\varphi):=(\overline{-1})^{\frac{n(n-1)}{2}} \operatorname{det}(\varphi)$ and call $d(\varphi)$ the discriminant of $\varphi$.

Finally we will need the Clifford invariant $c(\varphi) \in{ }_{2} \operatorname{Br}(K)$ of $\varphi$ as defined in [Lam05, chapter $\mathrm{V}$, (3.12)] (there $c$ is called the Witt invariant). Here $\operatorname{Br}(K)$ denotes the Brauer group of $K$ and ${ }_{2} \operatorname{Br}(K)$ is its subgroup of elements of order $\leq 2$. For $a, b \in K^{*}$ we define $\left(\frac{a, b}{K}\right)$ to be the quaternion algebra over $K$ with basis $1, i, j, i j$ such that $i^{2}=a, j^{2}=b$, and $i j=-j i$. Depending on the context $\left(\frac{a, b}{K}\right)$ can also stand for its image in $\operatorname{Br}(K)$. In these cases we also allow the notation $\left(\frac{\bar{a}, \bar{b}}{K}\right)$.

\section{Annihilating Polynomials}

3.1 Definition. Let $R$ be a unitary, commutative ring, and let $\iota: \mathbb{Z} \rightarrow R$ be the canonical homomorphism defined by $\iota(1)=1_{R}$. A polynomial $P=a_{n} X^{n}+\cdots+a_{1} X+a_{0} \in \mathbb{Z}[X]$ is called annihilating polynomial of an element $x \in R$ if

$$
P(x):=\iota\left(a_{n}\right) x^{n}+\cdots+\iota\left(a_{1}\right) x+\iota\left(a_{0}\right)=0 \in R .
$$

Usually we will omit the $\iota$ and simply write $a x:=\iota(a) x$ for $a \in \mathbb{Z}$ and $x \in R$.

3.2 Remark. It is clear that 0 and annihilating polynomials of degree 0 do not reveal a lot of information about an element $x \in R$. But we admit those polynomials for reasons of completeness and since we want the set of all annihilating polynomials of $x$ to form an ideal.

Recall that a group of exponent 2 is always Abelian. Henceforth we will consider such a group $G$ and its group ring $\mathbb{Z}[G]$. In [Hur89] Hurrelbrink describes how to construct annihilating polynomials for a given element of $\mathbb{Z}[G]$. He considers the set of ring homomorphisms $\operatorname{Hom}(\mathbb{Z}[G], \mathbb{Z})$ 
and notes that for any $f \in \mathbb{Z}[G]$, since $G$ is a torsion group, the set

$$
S_{f}:=\{\chi(f) \mid \chi \in \operatorname{Hom}(\mathbb{Z}[G], \mathbb{Z})\} \subset \mathbb{Z}
$$

is finite. Hence it is possible to define the polynomial

$$
P_{f}:=\prod_{\chi(f) \in S_{f}}(X-\chi(f)) \in \mathbb{Z}[X] .
$$

It follows from [Hur89, Theorem 1.3] that $P_{f}$ is an annihilating polynomial of $f$.

For the rest of this section let $J \subset \mathbb{Z}[G]$ be an ideal such that the quotient ring $R:=\mathbb{Z}[G] / J$ has only 2-torsion. Rings of this form are of special interest to us since both the Witt-Grothendieck Ring and the Witt Ring of a field can be obtained like this. In [KRW72] Knebusch, Rosenberg and Ware studied those rings and called them Witt Rings for G (see [KRW72, Definition 3.12 \& Theorem 3.9]). In the following we will examine the possible shapes of annihilating polynomials for an element $x \in R$.

3.3 Proposition. Let $R$ be a Witt Ring for a group $G$ of exponent 2, and let $Q_{x}$ be a greatest common divisor of all annihilating polynomials of $x \in R$. Then $Q_{x}$ can be chosen to be monic and a product of linear factors. Furthermore $Q_{x}(x)$ is a 2-torsion element of $R$.

Proof. By the definition of $R$ there exists a canonical projection $\pi: \mathbb{Z}[G] \rightarrow R$. Let $f \in \mathbb{Z}[G]$ with $\pi(f)=x$. We have seen that the polynomial $P_{f}$, as defined in (1), annihilates $f$. Hence, since $\pi$ is a ring homomorphism, $P_{f}$ is also an annihilating polynomial of $x$. Now $Q_{x}$ divides $P_{f}$ and hence can be chosen to be monic and a product of linear factors.

Since $\mathbb{Z}[X]$ is Noetherian, and since the set of all annihilating polynomials of $x$ forms an ideal, there exist annihilating polynomials $P_{1}, \ldots, P_{r} \in \mathbb{Z}[X]$, elements $\lambda_{1}, \ldots, \lambda_{r} \in \mathbb{Z}[X]$, and an integer $m \in \mathbb{Z}$ such that

$$
m Q_{x}=\lambda_{1} P_{1}+\cdots+\lambda_{r} P_{r}
$$

It follows that $m Q_{x}$ is an annihilating polynomial of $x$. Thus and by the definition of a Witt Ring for $G$ the element $Q_{x}(x)$ is 2-torsion.

3.4 Definition. Let $R$ be a Witt Ring for a group $G$ of exponent 2 , and let $x \in R$.

(1) The monic polynomial $Q_{x}$ from the previous proposition is called the embracing polynomial of $x$.

(2) The annihilating ideal of $x$ is the ideal $\operatorname{Ann}_{x} \subset \mathbb{Z}[X]$ consisting of all annihilating polynomials of $x$.

3.5 Remark. The specific name "embracing polynomial" was chosen in view of the fact that $\operatorname{Ann}_{x} \subset\left(Q_{x}\right)$ for $x \in R$, and $\left(Q_{x}\right)$ is the unique minimal principal ideal that contains $\operatorname{Ann}_{x}$. $\triangle$

We would like to find a method to explicitly calculate the embracing polynomial. To this end we consider the set of ring homomorphisms $\operatorname{Hom}(R, \mathbb{Z})$ and for $x \in R$ define the signature set

$$
S_{x}:=\{\chi(x) \mid \chi \in \operatorname{Hom}(R, \mathbb{Z})\} .
$$


Since every homomorphism $R \rightarrow \mathbb{Z}$ is induced by a homomorphism $\mathbb{Z}[G] \rightarrow \mathbb{Z}$ it follows from the above that $S_{x}$ is finite. Therefore we can define the signature polynomial

$$
P_{x}^{\text {sign }}:=\prod_{\chi(x) \in S_{x}}(X-\chi(x)) \in \mathbb{Z}[X] .
$$

If $\operatorname{Hom}(R, \mathbb{Z})=\varnothing$ then $P_{x}^{\text {sign }}$ is the empty product and hence equal to 1 .

3.6 Lemma. Consider a Witt Ring $R$ for a group $G$ of exponent 2 such that $\operatorname{Hom}(R, \mathbb{Z}) \neq \varnothing$. Then the torsion subgroup $R_{t}=\bigcap_{\chi \in \operatorname{Hom}(R, \mathbb{Z})} \operatorname{ker}(\chi)$.

Proof. Let $\operatorname{Nil}(R)$ denote the set of nilpotent elements of $R$. First we note that $\operatorname{Hom}(R, \mathbb{Z}) \neq \varnothing$ implies $R_{t} \neq R$. Hence by [KRW72, Theorem 3.9, Propositions $3.15 \& 3.16$ ] we must have $R_{t}=\operatorname{Nil}(R)$, which by [KRW72, Proposition 3.4] is equivalent to saying that no maximal ideal of $R$ is a minimal prime ideal.

Let $\pi: \mathbb{Z}[G] \rightarrow R$ be the canonical projection, and let $J \subset \mathbb{Z}[G]$ be the ideal such that $R=$ $\mathbb{Z}[G] / J$. Consider a minimal prime ideal $\mathfrak{p} \subset R$. Then $\mathfrak{p}^{\prime}=\pi^{-1}(\mathfrak{p})$ is a prime ideal of $\mathbb{Z}[G]$ containing $J$. It follows from [KRW72, Theorem 3.9.(i)] that $\mathfrak{p}^{\prime}$ is minimal. Thus by [KRW72, Lemma 3.1] there exists a $\chi^{\prime} \in \operatorname{Hom}(\mathbb{Z}[G], \mathbb{Z})$ such that $\mathfrak{p}^{\prime}=\operatorname{ker}\left(\chi^{\prime}\right)$. Now, since $J \subset \mathfrak{p}^{\prime}, \chi^{\prime}$ induces a ring homomorphism $\chi \in \operatorname{Hom}(R, \mathbb{Z})$ and $\mathfrak{p}=\operatorname{ker}(\chi)$. Hence we obtain

$$
R_{t}=\operatorname{Nil}(R)=\bigcap_{\mathfrak{p} \in \operatorname{Min}(R)} \mathfrak{p}=\bigcap_{\chi \in \operatorname{Hom}(R, \mathbb{Z})} \operatorname{ker}(\chi),
$$

where $\operatorname{Min}(R) \subset \operatorname{Spec}(R)$ denotes the set of minimal prime ideals.

3.7 Proposition. If $R$ is a Witt Ring for a group $G$ of exponent 2 , and if $x \in R$, then the embracing polynomial $Q_{x}$ is equal to the signature polynomial $P_{x}^{\text {sign }}$.

Proof. By [KRW72, Theorem 3.9, Propositions $3.15 \& 3.16$ ] there are the two possible cases $R_{t}=\operatorname{Nil}(R)$ and $R_{t}=R$. If $R_{t}=\operatorname{Nil}(R)$, then by the previous lemma $R_{t}=\bigcap_{\chi \in \operatorname{Hom}(R, \mathbb{Z})} \operatorname{ker}(\chi)$. If $R_{t}=R$, then we have $\operatorname{Hom}(R, \mathbb{Z})=\varnothing$ and we can set $\bigcap_{\chi \in \operatorname{Hom}(R, \mathbb{Z})} \operatorname{ker}(\chi)=R$. In both cases the element $x \in R$ is torsion if and only if $\chi(x)=0$ for all $\chi \in \operatorname{Hom}(R, \mathbb{Z})$.

As $\chi\left(P_{x}^{\mathrm{sign}}(x)\right)=P_{x}^{\mathrm{sign}}(\chi(x))=0$ for all $\chi \in \operatorname{Hom}(R, \mathbb{Z})$, we deduce that $P_{x}^{\mathrm{sign}}(x)$ is torsion, i.e. there exists some $m \in \mathbb{N}$ such that $m P_{x}^{\text {sign }}$ is an annihilating polynomial of $x$. Hence by definition $Q_{x}$ divides $m P_{x}^{\text {sign }}$, and since $Q_{x}$ is monic it must also divide $P_{x}^{\text {sign }}$. But now $Q_{x}$ is a product of linear factors, and as $Q_{x}(x)$ is torsion we have $Q_{x}(\chi(x))=\chi\left(Q_{x}(x)\right)=0$ for all $\chi \in \operatorname{Hom}(R, \mathbb{Z})$. Therefore, since $\mathbb{Z}[X]$ is a unique factorisation domain, $\chi(x)$ is a root of $Q_{x}$ or equivalently $X-\chi(x)$ divides $Q_{x}$ for all $\chi \in \operatorname{Hom}(R, \mathbb{Z})$. It follows that $P_{x}^{\text {sign }}$ divides $Q_{x}$ and hence $Q_{x}=P_{x}^{\text {sign }}$.

3.8 Example. Let $K$ be a field with $\operatorname{char}(K) \neq 2$, then it is well-known that the WittGrothendieck Ring $\widehat{W}(K)$ and the Witt Ring $W(K)$ are both Witt Rings for the square class group $G:=K^{*} /\left(K^{*}\right)^{2}$ (see for example [Sch85, chapter 2, Theorem $9.1 \&$ Corollary 9.4]). For every field $K$ there exists the dimension homomorphism $\operatorname{dim}: \widehat{W}(K) \rightarrow \mathbb{Z}$. Hence by [KRW72, Proposition 3.15] we obtain $\widehat{W}(K)_{t}=\operatorname{Nil}(\widehat{W}(K))$. If $K$ is formally real, then there exists at least one signature homomorphism $W(K) \rightarrow \mathbb{Z}$, whence we again obtain $W(K)_{t}=\operatorname{Nil}(W(K))$. 
If $K$ is not formally real, then there does not exist a ring homomorphism $W(K) \rightarrow \mathbb{Z}$. Thus it follows from [KRW72, Proposition 3.16] that $W(K)_{t}=W(K)$.

Let $\varphi$ be a quadratic form of dimension $n$ over a field $K$. First we assume that $K$ is not formally real. In this case it is easy to explicitly give the embracing polynomial of both $[\varphi] \in \widehat{W}(K)$ and $\{\varphi\} \in W(K)$. Recall that the dimension homomorphism $\operatorname{dim}: \widehat{W}(K) \rightarrow \mathbb{Z}$ is the only ring homomorphism $\widehat{W}(K) \rightarrow \mathbb{Z}$. Hence

$$
Q_{[\varphi]}=X-\operatorname{dim}(\varphi)=X-n \in \mathbb{Z}[X] .
$$

For the Witt Ring we have $\operatorname{Hom}(W(K), \mathbb{Z})=\varnothing$, which implies

$$
Q_{\{\varphi\}}=1 .
$$

Indeed 1 divides every annihilating polynomial of $\{\varphi\}$, and furthermore, since $W(K)$ is a torsion ring, the element $1 \in W(K)$ is torsion.

Now let $K$ be formally real. In this case $\operatorname{Hom}(\widehat{W}(K), \mathbb{Z})$ consists of the signature homomorphisms and the dimension homomorphism whereas $\operatorname{Hom}(W(K), \mathbb{Z})$ only consists of the signature homomorphisms. Thus for any field $K$ there exists a bijection

$$
\operatorname{Hom}(W(K), \mathbb{Z}) \longrightarrow \operatorname{Hom}(\widehat{W}(K), \mathbb{Z}) \backslash\{\operatorname{dim}\}, \quad \chi \longmapsto \chi \circ \pi_{2},
$$

where $\pi_{2}: \widehat{W}(K) \rightarrow W(K)$ is the canonical projection. We obtain

$$
Q_{[\varphi]}= \begin{cases}Q_{\{\varphi\}} & \text { if } n \in S_{\{\varphi\}} \\ (X-n) Q_{\{\varphi\}} & \text { otherwise }\end{cases}
$$

3.9 Example. Let $K$ be a field, and let $\varphi$ be an excellent form of dimension $n \in \mathbb{N}_{0}$ over $K$ (for the definition of excellent forms refer to [Kne77]). Denote by $n_{0}=n, n_{1}, \ldots, n_{h} \in \mathbb{N}_{0}$ the dimensions of the higher anisotropic kernels of $\varphi$. In [Rüh08] it is shown that the polynomial

$$
\mathbb{Z}[X] \ni E_{n}:= \begin{cases}X\left(X^{2}-n_{h-1}^{2}\right) \cdots\left(X^{2}-n_{1}^{2}\right)\left(X^{2}-n^{2}\right) & \text { for } n \text { even, } \\ \left(X^{2}-1\right)\left(X^{2}-n_{h-1}^{2}\right) \cdots\left(X^{2}-n_{1}^{2}\right)\left(X^{2}-n^{2}\right) & \text { for } n \text { odd }\end{cases}
$$

annihilates $[\varphi]$. We can now use the previous results to show that $K$ and $\varphi$ can be chosen such that $\operatorname{Ann}_{[\varphi]}=\operatorname{Ann}_{\{\varphi\}}=\left(E_{n}\right)$. This is clear if $n=0$, since $\operatorname{Ann}_{0}=(X)$ over any formally real field $K$.

Let $L$ be a Euclidean field, and let $k \in \mathbb{N}_{0}$. Then $K:=L\left(\left(t_{0}\right)\right)\left(\left(t_{1}\right)\right) \ldots\left(\left(t_{k}\right)\right)$, where $t_{i}$ is transcendental over $L\left(\left(t_{0}\right)\right) \ldots\left(\left(t_{i-1}\right)\right)$ for $i=0, \ldots, k$, is Pythagorean, $W(K)$ is torsion free, and there exist $2^{k+1}$ signature homomorphisms $W(K) \rightarrow \mathbb{Z}$ (see [Lam05, chapter VIII, proposition 4.11]). More precisely, if $U \subset\{0, \ldots, k\}$ is any subset, then there exists a unique signature homomorphism $\chi$ such that $\chi\left(\left\{\left\langle t_{i}\right\rangle\right\}\right)=1$ if $i \in U$ and $\chi\left(\left\{\left\langle t_{i}\right\rangle\right\}\right)=-1$ otherwise. Consider the form $\tau:=\left\langle\left\langle t_{1}, \ldots, t_{k}\right\rangle\right\rangle$ over $K$. Then

$$
S_{\left[t_{0} \tau\right]}=S_{\left\{t_{0} \tau\right\}}=\left\{-2^{k}, 0,2^{k}\right\} .
$$


We proceed by induction on $k$ to show that for all $n \in \mathbb{N}$ with $2^{k-1}<n \leq 2^{k}$ there exists an excellent $n$-dimensional subform $\varphi$ of $t_{0} \tau$ that has $\operatorname{Ann}_{[\varphi]}=\operatorname{Ann}_{\{\varphi\}}=\left(E_{n}\right)$. If $k=0$, then $\tau=\langle 1\rangle$ and $\varphi \cong\left\langle t_{0}\right\rangle$, and the claim follows immediately.

We continue with the case $k>0$. Then $n_{1}=2^{k}-n<2^{k-1}$. If $n_{1}=0$, then $\varphi \cong t_{0} \tau, h=1$, and $\operatorname{Ann}_{[\varphi]}=\operatorname{Ann}_{\{\varphi\}}=\left(E_{n}\right)=\left(X\left(X^{2}-n^{2}\right)\right)$. Otherwise let $l \in \mathbb{N}_{0}$ be minimal such that $n_{1} \leq 2^{l}$. By the induction hypothesis there exists an $n_{1}$-dimensional excellent subform $\psi$ of $t_{0}\left\langle\left\langle t_{1}, \ldots, t_{l}\right\rangle\right\rangle$ over $L\left(\left(t_{0}\right)\right)\left(\left(t_{1}\right)\right) \ldots\left(\left(t_{l}\right)\right) \subset K$ with $S_{[\psi]}=S_{\{\psi\}}=\left\{n_{1},-n_{1}, n_{2},-n_{2}, \ldots, n_{h},-n_{h}\right\}$. If $\varphi$ is a form over $K$ such that $\varphi \perp \psi_{K} \cong t_{0} \tau$, then $\varphi$ is excellent and

$$
\chi(\{\varphi\})+\chi\left(\left\{\psi_{K}\right\}\right)= \begin{cases}2^{k} & \text { if } \chi\left(\left\{\left\langle t_{i}\right\rangle\right\}\right)=1 \text { for } i=0, \ldots, k, \\ -2^{k} & \text { if } \chi\left(\left\{\left\langle t_{0}\right\rangle\right\}\right)=-1 \text { and } \operatorname{sign}\left(\left\{\left\langle t_{i}\right\rangle\right\}\right)=1 \\ & \text { for } i=1, \ldots, k, \\ 0 & \text { otherwise, }\end{cases}
$$

for $\chi \in \operatorname{Hom}(W(K), \mathbb{Z})$. Hence it follows easily that $S_{[\varphi]}=S_{\{\varphi\}}=\{n,-n\} \cup S_{\left\{\psi_{K}\right\}}$, which concludes the induction.

Let $x \in R$ be any element. We conclude this section by developing a rough concept of the structure of $\mathrm{Ann}_{x}$. More specifically we want to construct a full set of generators for $\operatorname{Ann}_{x}$.

First we need to fix some notation. For a polynomial $P=a_{n} X^{n}+\cdots+a_{1} X+a_{0} \in \mathbb{Z}[X]$ with $a_{n} \neq 0$ we denote by $\operatorname{lc}(P):=a_{n}$ the leading coefficient of $P$. Furthermore for $a \in \mathbb{Z} \backslash\{0\}$ let $v_{2}(a)$ denote the 2 -adic valuation of $a$, i.e. $a=2^{v_{2}(a)} b$ with $b \in \mathbb{Z}$ odd. We set $v_{2}(0):=+\infty$.

Now let $k_{0} \in \mathbb{N}_{0}$ be minimal such that $2^{k_{0}} Q_{x}$ is an annihilating polynomial of $x$. Set $P_{0}:=2^{k_{0}} \in$ $\mathbb{Z}[X]$. If $k_{0} \neq 0$ we continue by compiling a sequence of polynomials $P_{1}, \ldots, P_{r} \in \mathbb{Z}[X]$ together with a sequence of natural numbers $k_{1}, \ldots, k_{r} \in \mathbb{N}_{0}$ as follows:

Assume that for $i \geq 1$ the polynomial $P_{i-1}$ and $k_{i-1} \in \mathbb{N}$ have already been chosen. Then there exists a polynomial $P_{i} \in \mathbb{Z}[X]$ of minimal degree with the following three properties:

(GP1) The product $P_{i} Q_{x}$ is an annihilating polynomial of $x$.

(GP2) We have $\operatorname{lc}\left(P_{i}\right)=2^{k_{i}}$ with $k_{i} \in \mathbb{N}_{0}$ and $k_{i}<k_{i-1}$.

(GP3) If $P^{\prime} \in \mathbb{Z}[X]$ is another polynomial with $\operatorname{deg}\left(P^{\prime}\right)=\operatorname{deg}\left(P_{i}\right)$ and $\operatorname{lc}\left(P^{\prime}\right)=2^{l}$, then $k_{i} \leq l$.

If $k_{i}=0$, then $r=i$ and the process stops. Otherwise we continue with the construction of $P_{i+1}$.

The construction will stop after a finite number of steps since there does exist a monic annihilating polynomial of $x$. Indeed let $\pi: \mathbb{Z}[G] \rightarrow R$ be the canonical projection and $f \in \mathbb{Z}[G]$ such that $\pi(f)=x$. Then the $P_{f}$ as defined in (1) is a monic annihilating polynomial of $x$.

We note that property (GP2) implies $\operatorname{deg}\left(P_{i}\right)>\operatorname{deg}\left(P_{i-1}\right)$ for $i=1, \ldots, r$.

3.10 Remark. A more general way of choosing the generators of an ideal in $\mathbb{Z}[X]$ has been introduced by G. Szekeres in [Sze52]. In fact it will follow from our results further down that in our particular case Szekeres' method and the method introduced above coincide. 
Next we show that there exist monic polynomials $Q_{1}, \ldots, Q_{r}$ such that $P_{i}=2^{k_{i}} Q_{i}$.

3.11 Lemma. If $x \in R$ and $P \in \mathbb{Z}[X]$ with $P Q_{x} \in \operatorname{Ann}_{x}, \operatorname{deg}(P)=d \in \mathbb{N}_{0}$, and $v_{2}(\operatorname{lc}(P))=l$, then there exists a $P^{\prime} \in \mathbb{Z}[X]$ such that $P^{\prime} Q_{x} \in \operatorname{Ann}_{x}, \operatorname{deg}\left(P^{\prime}\right)=d$, and $\operatorname{lc}\left(P^{\prime}\right)=2^{l}$.

Proof. Let $\operatorname{lc}(P)=m 2^{l}$ with $m$ odd, and let $k_{0} \in \mathbb{N}_{0}$ be minimal such that $2^{k_{0}} Q_{x} \in \operatorname{Ann}_{x}$. It is clear that $2^{k_{0}} X^{d} Q_{x}$ lies in $\operatorname{Ann}_{x}$. Furthermore $\operatorname{gcd}\left(m 2^{l}, 2^{k_{0}}\right)=2^{l^{\prime}}$ with $l^{\prime}=\min \left(l, k_{0}\right)$. Let $\alpha, \beta \in \mathbb{Z}$ such that $\alpha m 2^{l}+\beta 2^{k_{0}}=2^{l^{\prime}}$. If we set

$$
P^{\prime}:=2^{l-l^{\prime}}\left(\alpha P+\beta 2^{k_{0}} X^{d}\right),
$$

then $\operatorname{deg}\left(P^{\prime}\right)=d, \operatorname{lc}\left(P^{\prime}\right)=2^{l}$, and $P^{\prime} Q_{x}$ is an annihilating polynomial of $x$.

For $x \in R$ and $d \in \mathbb{N}_{0}$ we define

$$
\operatorname{minv}_{x, 2}(d):=\min \left\{v_{2}(\operatorname{lc}(P)) \mid P \in \mathbb{Z}[X], \operatorname{deg}(P)=d, P Q_{x} \in \operatorname{Ann}_{x}\right\} .
$$

From the previous lemma we can now deduce, that in the above construction of the $P_{i}$ the requirement that $P_{i}$ must have a 2 -power leading coefficient is not really a constraint.

3.12 Corollary. Let $x \in R$ and $P \in \mathbb{Z}[X]$ with $P Q_{x} \in \operatorname{Ann}_{x}$ and $\operatorname{deg}(P)=d \in \mathbb{N}_{0}$. If $i \in\{0, \ldots, r\}$ is maximal with $\operatorname{deg}\left(P_{i}\right) \leq d$, then $v_{2}(\operatorname{lc}(P)) \geq k_{i}$. In particular $\operatorname{minv}_{x, 2}(d)=k_{i}$.

3.13 Lemma. Let $P=a_{d} X^{d}+\cdots+a_{1} X+a_{0} \in \mathbb{Z}[X], \operatorname{deg}(P)=d \in \mathbb{N}_{0}$, such that $P Q_{x}$ is an annihilating polynomial of $x \in R$. Then $v_{2}\left(a_{i}\right) \geq \operatorname{minv}_{x, 2}(d)$ for all $i=0, \ldots, d$.

Proof. We proceed by induction on $d$. If $d=0$, then $P=m 2^{l}$ with $m \in \mathbb{Z}$ odd and $l \geq k_{0}=$ $\operatorname{minv}_{x, 2}(0)$, which concludes the proof in this case.

Now let $d>0$, and let $i \in\{0, \ldots, r\}$ be maximal such that $\operatorname{deg}\left(P_{i}\right) \leq d$. Then by the previous corollary we have $\operatorname{minv}_{x, 2}(d)=k_{i}$. We need to distinguish between the two cases $P=P_{i}$ and $P \neq P_{i}$, and we first need to assume that $P=P_{i}$. In this case we trivially have $v_{2}\left(a_{d}\right) \geq k_{i}$. Since $d>0$, we must have $i>0$. Set $d_{i-1}:=\operatorname{deg}\left(P_{i-1}\right)$. Then

$$
P^{\prime}:=2^{k_{i-1}-k_{i}} P_{i}-X^{d-d_{i-1}} P_{i-1}
$$

has degree strictly smaller than $d$, and $P^{\prime} Q_{x}$ is an annihilating polynomial of $x$. Let $P^{\prime}=$ $b_{d-1} X^{d-1}+\cdots+b_{1} X+b_{0}$ and $P_{i-1}=c_{d_{i-1}} X^{d_{i-1}}+\cdots+c_{1} X+c_{0}$ with $b_{0}, \ldots, b_{d-1}, c_{0}, \ldots, c_{d_{i-1}} \in$ $\mathbb{Z}$. Set $c_{j}=0$ for $j<0$, and recall that $v_{2}(0)=+\infty$. Since $\operatorname{deg}\left(P^{\prime}\right), \operatorname{deg}\left(P_{i-1}\right)<\operatorname{deg}\left(P_{i}\right)$ the induction hypothesis states that $v_{2}\left(b_{j}\right) \geq k_{i-1}$ and $v_{2}\left(c_{j}\right) \geq k_{i-1}$ for all $j$. As

$$
b_{j}=2^{k_{i-1}-k_{i}} a_{j}-c_{d_{i-1}-d+j}
$$

for $j=0, \ldots, d-1$, it follows that $v_{2}\left(2^{k_{i-1}-k_{i}} a_{j}\right)=k_{i-1}-k_{i}+v_{2}\left(a_{j}\right) \geq k_{i-1}$ and hence $v_{2}\left(a_{j}\right) \geq k_{i}$.

Now we consider the case $P \neq P_{i}$. By corollary 3.12 we have $v_{2}\left(a_{d}\right)=v_{2}(\operatorname{lc}(P)) \geq k_{i}$. Hence there exists an $a \in \mathbb{Z}$ such that $a_{d}=a 2^{k_{i}}$. Set $d_{i}:=\operatorname{deg}\left(P_{i}\right)$. Then the polynomial

$$
P^{\prime \prime}:=P-a X^{d-d_{i}} P_{i}
$$


has degree strictly smaller than $\operatorname{deg}(P)$, and $P^{\prime \prime} Q_{x}$ lies in $\operatorname{Ann}_{x}$. Let $b_{0}, \ldots, b_{d-1}, c_{0}, \ldots, c_{d_{i}} \in \mathbb{Z}$ with $P^{\prime \prime}=b_{d-1} X^{d-1}+\cdots+b_{1} X+b_{0}$ and $P_{i}=c_{d_{i}} X^{d_{i}}+\cdots+c_{1} X+c_{0}$. If we assume that $c_{j}=0$ for $j<0$, then $b_{j}=a_{j}-a c_{d_{i}-d+j}$ for $j=0, \ldots, d-1$. Now the induction hypothesis states, that if $\operatorname{deg}\left(P^{\prime \prime}\right) \geq \operatorname{deg}\left(P_{i}\right)$, we have $v_{2}\left(b_{j}\right) \geq k_{i}$, and if $\operatorname{deg}\left(P^{\prime \prime}\right)<\operatorname{deg}\left(P_{i}\right)$, then we have $v_{2}\left(b_{j}\right) \geq k_{i-1}>k_{i}$ for $j=0, \ldots, d-1$. Furthermore by the induction hypothesis or what we have shown previously $v_{2}\left(c_{j}\right) \geq k_{i}$ for all $j$. Hence we obtain $v_{2}\left(a_{j}\right) \geq k_{i}$ for $j=0, \ldots, d-1$.

Thus we can indeed choose $P_{i}=2^{k_{i}} Q_{i}$ with $Q_{i} \in \mathbb{Z}[X]$ monic for $i=0, \ldots, r$.

3.14 Proposition. Let $R$ be a Witt Ring for a group $G$ of exponent 2 , and let $x \in R$. If $Q_{0}=1, Q_{1}, \ldots, Q_{r} \in \mathbb{Z}[X]$ and $k_{0}, \ldots, k_{r}=0 \in \mathbb{Z}[X]$ are sequences as constructed above, then

$$
\mathcal{B}:=\left\{2^{k_{0}} Q_{x}, 2^{k_{1}} Q_{1} Q_{x}, \ldots, 2^{k_{r-1}} Q_{r-1} Q_{x}, Q_{r} Q_{x}\right\}
$$

is a full set of generators for $\mathrm{Ann}_{x}$.

Proof. Let $B$ be the ideal of $\mathbb{Z}[X]$ generated by the elements of $\mathcal{B}$. It is clear that $B \subset \operatorname{Ann}_{x}$. It remains to show that the inclusion $\mathrm{Ann}_{x} \subset B$ holds as well. Let $0 \neq P \in \mathbb{Z}[X]$ with $P Q_{x} \in \mathrm{Ann}_{x}$. We proceed by induction on $d:=\operatorname{deg}(P)$.

If $0=\operatorname{deg}(P)$, then $P=2^{l} m$ with $m \in \mathbb{Z}$ odd. By lemma 3.13 we have $l \geq \operatorname{minv}_{x, 2}(0)=k_{0}$. Hence there exists some integer $b \in \mathbb{Z}$ such that $P=b 2^{k_{0}}$ and therefore $P Q_{x} \in B$.

Now let $d>0$, and let $i \in\{0, \ldots, r\}$ be maximal such that $d \geq \operatorname{deg}\left(P_{i}\right)$. Let $m=\operatorname{lc}(P)$. By lemma 3.13 we have $v_{2}(m) \geq \operatorname{minv}_{x, 2}(d)=k_{i}$. Again there exists an integer $b \in \mathbb{Z}$ such that the polynomial

$$
P^{\prime}:=P-b X^{d-\operatorname{deg}\left(P_{i}\right)} P_{i}
$$

has degree strictly smaller than $d$, and $P^{\prime} Q_{x}$ is an annihilating polynomial of $x$. By the induction hypothesis $P^{\prime}$ lies in $B$, and hence the same holds for $P$.

Thus we have shown that $\mathrm{Ann}_{x}=B$.

3.15 Remark. It is possible to generalise Lemma 3.13 to the case where we consider an arbitrary ideal $A \subset R[X]$ with $R$ a principal ideal domain. Let $Q$ be a greatest common divisor of the elements of $A$. There exists a sequence of polynomials $P_{0}, \ldots, P_{r} \in R[X]$ with leading coefficients $m_{0}=\operatorname{lc}\left(P_{0}\right), \ldots, m_{r}=\operatorname{lc}\left(P_{r}\right) \in R$ such that $P_{0}=m_{0}, \operatorname{deg}\left(P_{i-1}\right)<\operatorname{deg}\left(P_{i}\right)$ and $m_{i} \mid m_{i-1}$ with $m_{i} \neq m_{i-1}$ for $i=1, \ldots, r$, and $\left\{m_{0} Q, P_{1} Q, \ldots, P_{r} Q\right\}$ is a full set of generators of $A$. Furthermore $P_{0}, \ldots, P_{r}$ have been chosen such that for any $P \in R[X]$ with $P Q \in A$ and $i \in\{0, \ldots, r\}$ maximal with $\operatorname{deg}(P) \geq \operatorname{deg}\left(P_{i}\right)$ we have $m_{i} \mid \operatorname{lc}(P)$. In addition we can choose $P_{i}=m_{i} Q_{i}$ with $Q_{i} \in \mathbb{Z}[X]$ monic. This is a direct consequence of Szekeres' observations in [Sze52] (see also [Réd67, Theorem $285, \S 120]$ ). Among other things this implies that we must have $m_{r}=1$.

3.16 Remark. Proposition 3.14 raises two main questions:

(1) Are there any restrictions on the differences

(a) $\operatorname{deg}\left(Q_{i}\right)-\operatorname{deg}\left(Q_{i-1}\right) \geq 1$ or

(b) $k_{i-1}-k_{i} \geq 1$ ? 
(2) In case of a positive answer to question (1).(a) or (1).(b), what do the restrictions look like and how can they be related to properties of $x$ ?

At least to question (1).(a) we can already give a positive and quite satisfying answer. In fact it is possible to generalise the induction basis of the proof for [OG97, Lemma 2.2] to our more general case. Thus we can deduce that $\operatorname{deg}\left(Q_{i}\right)-\operatorname{deg}\left(Q_{i-1}\right) \in\{1,2\}$. This raises the more specific question

$\left(2^{\prime}\right)$.(a) What property of $x$ determines whether the difference $\operatorname{deg}\left(Q_{i}\right)-\operatorname{deg}\left(Q_{i-1}\right)$ equals 1 or 2 ?

\section{Local Fields}

During all of this section let $K$ be a local field with finite residue field. It is well known, that over such a field the third power $I^{3}(K)$ of the fundamental ideal $I(K)$ of $W(K)$ vanishes. This implies that equivalence classes of quadratic forms over $K$ are completely classifiable by the dimension modulo 2, the discriminant $d$, and the Clifford invariant $c$. It follows that isometry classes of forms over $K$ can be completely classified with the help of the dimension homomorphism, the discriminant, and the Clifford invariant. By means of calculations with these invariants we would like to determine for a quadratic form $\varphi$ over $K$ full sets of generators for $\mathrm{Ann}_{[\varphi]}$ and $\mathrm{Ann}_{\{\varphi\}}$.

Let $\varphi$ be an $n$-dimensional quadratic form over $K$. We start with a few observations on $\operatorname{Ann}_{[\varphi]}$. Since $K$ is not formally real, we have seen that $Q_{[\varphi]}=X-n$. If $\varphi \cong n$, then of course $\operatorname{Ann}_{[\varphi]}=(X-n)$. In the other case we will show that there exists a linear factor $X-r \in \mathbb{Z}[X]$ such that $(X-r)(X-n) \in \operatorname{Ann}_{[\varphi]}$. Now we apply proposition 3.14. Since the level $s(K)$ of the local field $K$ is either 1, 2 or 4 , and since the equivalence class of $4 \times(\varphi \perp-n)$ lies in $I^{3}(K)$, it remains to check whether $2(X-n) \in \mathrm{Ann}_{[\varphi]}$ or not. We obtain

$$
\operatorname{Ann}_{[\varphi]}=(2(X-n),(X-r)(X-n))
$$

$$
\text { or } \quad \operatorname{Ann}_{[\varphi]}=(4(X-n),(X-r)(X-n)) \text {. }
$$

To find a full set of generators for $\mathrm{Ann}_{\{\varphi\}}$ is more complicated, since we have $Q_{\{\varphi\}}=1$. First we exclude the trivial case $\varphi \sim r$ for some $r \in \mathbb{Z}$, since in that case $\operatorname{Ann}_{\{\varphi\}}=(2 s(K), X-r)$. In the other case we consider [OG97, Lemma 3.4] which implies that $X(X+2) \in \mathbb{Z}[X]$ is an annihilating polynomial for every equivalence class of even-dimensional quadratic forms over $K$. This fact is also a consequence of the calculations concerning the Clifford invariant that we will undertake below. It follows that $(X+1)(X+3)$ is an annihilating polynomial for every equivalence class of odd-dimensional quadratic forms. Now if $s(K)=1$, then $2=0 \in W(K)$ and we can conclude that

$$
\operatorname{Ann}_{\{\varphi\}}= \begin{cases}(2, X(X+2))=\left(2, X^{2}\right) & \text { for } \operatorname{dim}(\varphi) \text { even } \\ (2,(X+1)(X+3))=\left(2,(X+1)^{2}\right) & \text { for } \operatorname{dim}(\varphi) \text { odd }\end{cases}
$$


If $s(K)=2$ and $\operatorname{dim}(\varphi)$ is even, then we only need to check whether the polynomial $2 X$ lies in $\operatorname{Ann}_{\{\varphi\}}$, since $2 X \in \mathrm{Ann}_{\{\varphi\}}$ if and only if $2(X+2) \in \mathrm{Ann}_{\{\varphi\}}$. So

$$
\operatorname{Ann}_{\{\varphi\}}=(4,2 X, X(X+2))=\left(4,2 X, X^{2}\right) \quad \text { or } \quad \operatorname{Ann}_{\{\varphi\}}=(4, X(X+2)) .
$$

For the odd-dimensional case it follows that

$$
\operatorname{Ann}_{\{\varphi\}}=\left(4,2(X+1),(X+1)^{2}\right) \quad \text { or } \quad \operatorname{Ann}_{\{\varphi\}}=(4,(X+1)(X+3)) .
$$

Finally, in the case that $s(K)=4$ and $\operatorname{dim}(\varphi)$ is even, it suffices to consider the polynomials $2 X, 2(X+2)$, and $4 X$. Indeed $2 X, 2(X+2) \in \operatorname{Ann}_{\{\varphi\}}$ would imply $4 \in \operatorname{Ann}_{\{\varphi\}}$, which is impossible since $s(K)>2$. Furthermore, if $4 X \in \operatorname{Ann}_{\{\varphi\}}$, then it follows from $8 \times\langle 1\rangle \sim 0$ that also $4(X+k) \in \operatorname{Ann}_{\{\varphi\}}$ for all $k \in 2 \mathbb{Z}$. But in fact we will see that either $2 X \in \operatorname{Ann}_{\{\varphi\}}$ or $2(X+2) \in \operatorname{Ann}_{\{\varphi\}}$ for any form $\varphi$ over $K$. Hence we have exactly the two cases

$$
\operatorname{Ann}_{\{\varphi\}}=\left(8,2 X, X^{2}\right) \quad \text { or } \quad \operatorname{Ann}_{\{\varphi\}}=\left(8,2(X+2),(X+2)^{2}\right) .
$$

For the case that $\varphi$ is odd-dimensional we obtain

$$
\operatorname{Ann}_{\{\varphi\}}=\left(8,2(X+1),(X+1)^{2}\right) \quad \text { or } \quad \operatorname{Ann}_{\{\varphi\}}=\left(8,2(X+3),(X+3)^{2}\right) .
$$

We will now begin our actual calculations. For an element $\{\varphi\}$ of $W(K)$ to be $0, \varphi$ must have even dimension, trivial discriminant, and trivial Clifford invariant. An element $x \in \widehat{W}(K)$ is 0 , if it has dimension 0 and its image in $W(K)$ vanishes. The calculations concerning the dimension are all trivial, and since the discriminant vanishes on the second power of the fundamental ideal, we will only need it below for our calculations concerning the Clifford invariant.

It is necessary that we establish certain formulas for the Clifford invariant. Let $\varphi$ and $\psi$ be quadratic forms over $K$. Then, by using the formulas (3.15) and (3.16) in [Lam05, chapter V, section3], we obtain

$$
c(\varphi \otimes \psi)=\left(\frac{d(\varphi), d(\psi)}{K}\right) \quad \text { for } \varphi \text { and } \psi \text { even-dimensional. }
$$

By the definition of the discriminant $d(r \times\langle 1\rangle)=(-1)^{\frac{r(r-1)}{2}}$, and it can easily be checked that $d(r \times\langle-1\rangle)=(-1)^{\frac{r(r+1)}{2}}=(-1)^{\frac{-r(-r-1)}{2}}$ for all $r \in \mathbb{N}_{0}$. To put it more shortly:

$$
d(r)=(-1)^{\frac{r(r-1)}{2}} \quad \forall r \in \mathbb{Z} .
$$

It is clear that $d(\varphi \perp \psi)=d(\varphi) d(\psi)$ for even-dimensional $\varphi$ and $\psi$. If $\varphi$ and $\psi$ have odd dimension, then we have $d(\varphi \perp \psi)=-d(\varphi) d(\psi)$. Let $a, b \in \mathbb{Z}$ and $n=\operatorname{dim}(\varphi)$ with $n \equiv a \equiv b(\bmod 2)$. If we combine (2) with our considerations about the discriminant we obtain

$$
c((\varphi \perp a) \otimes(\varphi \perp b))=\left(\frac{(-1)^{n} d(\varphi),-1}{K}\right)^{1+\frac{a(a-1)}{2}+\frac{b(b-1)}{2}}\left(\frac{-1,-1}{K}\right)^{\frac{a(a-1)}{2} \frac{b(b-1)}{2}}
$$

and

$$
c(2 \times(\varphi \perp a))=\left(\frac{(-1)^{n} d(\varphi),-1}{K}\right)\left(\frac{-1,-1}{K}\right)^{\frac{a(a-1)}{2}} .
$$


4.1 Example. We now consider the formulas (3) and (4) in a number of special cases that are of particular interest to us.

(1) Assume that $b \equiv a(\bmod 4)$ with $a \equiv n(\bmod 2)$. Then the table

\begin{tabular}{c|c|c}
\hline $\mathrm{a}$ & $\mathrm{n}$ & even \\
\hline$\equiv 0,1(\bmod 4)$ & $\left(\frac{d(\varphi),-1}{K}\right)$ & $\left(\frac{-d(\varphi),-1}{K}\right)$ \\
\hline$\equiv 2,3(\bmod 4)$ & $\left(\frac{-d(\varphi),-1}{K}\right)$ & $\left(\frac{d(\varphi),-1}{K}\right)$
\end{tabular}

shows the possible values of both $c((\varphi \perp a) \otimes(\varphi \perp b))$ and $c(2 \times(\varphi \perp a))$ depending on $n$ and $a$.

(2) Set $a=-r$ and $b=-s$ with $r \equiv s \equiv n(\bmod 2)$. This will be the case we have to consider in the context of signatures. Then we obtain the table

\begin{tabular}{c|c|c}
$\mathrm{s}$ & $\equiv 0,1(\bmod 4)$ & $\equiv 2,3(\bmod 4)$ \\
\hline$\equiv 0,1(\bmod 4)$ & $\left(\frac{d(\varphi),-1}{K}\right)$ & 1 \\
\hline$\equiv 2,3(\bmod 4)$ & 1 & $\left(\frac{-d(\varphi),-1}{K}\right)$
\end{tabular}

for $c((\varphi \perp-r) \otimes(\varphi \perp-s))$ with $n$ arbitrary. In particular

$$
c((\varphi \perp-r) \otimes(\varphi \perp-s))=\left(\frac{(-1)^{\frac{r(r-1)}{2}} d(\varphi),-1}{K}\right)
$$

for $r \equiv s(\bmod 4)$. Furthermore

$$
c(2 \times(\varphi \perp-r))=\left(\frac{(-1)^{\frac{r(r-1)}{2}} d(\varphi),-1}{K}\right) .
$$

(3) Consider the case $a=-n$ and $b=-r$ with $r \equiv n(\bmod 2)$. This case will come up in the context of isometry classes of quadratic forms. We obtain the surprisingly simple table

\begin{tabular}{c|c|c}
\hline $\mathrm{r}$ & $\equiv 0,1(\bmod 4)$ & $\equiv 2,3(\bmod 4)$ \\
\hline$\equiv 0,1(\bmod 4)$ & $\left(\frac{\operatorname{det}(\varphi),-1}{K}\right)$ & 1 \\
\hline$\equiv 2,3(\bmod 4)$ & 1 & $\left(\frac{\operatorname{det}(\varphi),-1}{K}\right)$
\end{tabular}


for $c((\varphi \perp-n) \otimes(\varphi \perp-r))$. Accordingly we have

$$
c(2 \times(\varphi \perp-n))=\left(\frac{\operatorname{det}(\varphi),-1}{K}\right)
$$

as a special case of equation (5)

Let $a \in K^{*}$. It is well-known that $\left(\frac{a,-1}{K}\right)=1 \in \operatorname{Br}(K)$ if and only if $\langle 1,1,-a\rangle$ is isotropic if and only if $a$ is a sum of two squares in $K$. Using this we can now translate the above tables into the following theorem.

4.2 Theorem. Let $K$ be a local field with finite residue field, and let $\varphi$ be a quadratic form over $K$ with $\operatorname{dim}(\varphi)=n \in \mathbb{N}_{0}$.

(1) If $\varphi \cong n$, then $\operatorname{Ann}_{[\varphi]}=(X-n) \subset \mathbb{Z}[X]$.

(2) If $\varphi \neq n$, then

$$
\operatorname{Ann}_{[\varphi]}= \begin{cases}\left(2(X-n),(X-n)^{2}\right) & \text { if } \operatorname{det}(\varphi) \text { is a sum of } \\ (4(X-n),(X-n+2)(X-n)) & \text { two squares in } K\end{cases}
$$

Next we formulate an analogous result for $\operatorname{Ann}_{\{\varphi\}}$. For the case $s(K)<4$ we note that $4 \times\langle a\rangle \sim 0$ for any $a \in K^{*}$. This implies $\langle\langle 1,-a\rangle\rangle \cong\langle\langle 1, a\rangle\rangle$. Therefore $\left(\frac{-a,-1}{K}\right)=\left(\frac{a,-1}{K}\right)$ and $\left(\frac{-1,-1}{K}\right)=1$ in $\operatorname{Br}(K)$.

Consider the case $s(K)=4$. Let $a \in K^{*}$. Assume that $\langle\langle 1, a\rangle\rangle$ and $\langle\langle 1,-a\rangle\rangle$ are both anisotropic. Since up to isometry there exists only one anisotropic quadratic form of dimension 4 over $K$, this implies $\langle\langle 1, a\rangle\rangle \sim\langle\langle 1,-a\rangle\rangle$. It follows that $4 \times\langle a\rangle \sim 0$, which is impossible since $s(K)=4$. Analogously it can be shown that not both $\langle\langle 1, a\rangle\rangle$ and $\langle\langle 1,-a\rangle\rangle$ can be hyperbolic. Hence either $a$ is a sum of two squares in $K$ or $-a$ is a sum of two squares in $K$.

4.3 Theorem. Let $K$ be a local field with finite residue field, and let $\varphi$ be a quadratic form over $K$ with $\operatorname{dim}(\varphi)=n \in \mathbb{N}_{0}$.

(1) If $\varphi \sim r$ with $r \in \mathbb{Z}$, then $\operatorname{Ann}_{\{\varphi\}}=(2 s(K), X-r) \subset \mathbb{Z}[X]$.

(2) If $\varphi \not r$ for all $r \in \mathbb{Z}$, and

(a) if $s(K)=1$, then

$$
\operatorname{Ann}_{\{\varphi\}}= \begin{cases}\left(2, X^{2}\right) & \text { if } n \text { is even } \\ \left(2,(X+1)^{2}\right) & \text { if } n \text { is odd. }\end{cases}
$$

(b) if $s(K)=2$, and

(i) if $n$ is even, then

$$
\operatorname{Ann}_{\{\varphi\}}= \begin{cases}\left(4,2 X, X^{2}\right) & \text { if } d(\varphi) \text { is a sum of } \\ (4, X(X+2)) & \text { two squares in } K\end{cases}
$$


(ii) if $n$ is odd, then

$$
\operatorname{Ann}_{\{\varphi\}}= \begin{cases}\left(4,2(X+1),(X+1)^{2}\right) & \text { if } d(\varphi) \text { is a sum of } \\ (4,(X-1)(X+1)) & \text { otherwise. }\end{cases}
$$

(c) if $s(K)=4$, and

(i) if $n$ is even, then

$$
\operatorname{Ann}_{\{\varphi\}}= \begin{cases}\left(8,2 X, X^{2}\right) & \text { if } d(\varphi) \text { is a sum of } \\ \left(8,2(X+2),(X+2)^{2}\right) & \text { two squares in } K, \\ \text { otherwise. }\end{cases}
$$

(ii) if $n$ is odd, then

$$
\operatorname{Ann}_{\{\varphi\}}= \begin{cases}\left(8,2(X-1),(X-1)^{2}\right) & \text { if } d(\varphi) \text { is a sum of } \\ & \text { two squares in } K, \\ \left(8,4(X+1),(X+1)^{2}\right) & \text { otherwise. }\end{cases}
$$

4.4 Definition. Let $K$ be a field. We set

$$
\begin{aligned}
& \operatorname{Ann}_{W(K)}^{(e)}:=\{P \in \mathbb{Z}[X] \mid P(\{\varphi\})=0 \forall\{\varphi\} \in W(K), \operatorname{dim}(\varphi) \text { even }\}, \\
& \operatorname{Ann}_{W(K)}^{(o)}:=\{P \in \mathbb{Z}[X] \mid P(\{\varphi\})=0 \forall\{\varphi\} \in W(K), \operatorname{dim}(\varphi) \text { odd }\}, \\
& \operatorname{Ann}_{W(K)}:=\operatorname{Ann}_{W(K)}^{(e)} \cap \operatorname{Ann}_{W(K)}^{(o)} .
\end{aligned}
$$

4.5 Corollary. Let $K$ be a local field with finite residue field.

(1) If $s(K)=1$, then

$$
\begin{aligned}
\operatorname{Ann}_{W(K)}^{(e)} & =\left(2, X^{2}\right), \\
\operatorname{Ann}_{W(K)}^{(o)} & =\left(2,(X+1)^{2}\right), \\
\operatorname{Ann}_{W(K)} & =\left(2, X^{2}(X+1)^{2}\right) .
\end{aligned}
$$

(2) If $s(K)=2$, then

$$
\begin{aligned}
\operatorname{Ann}_{W(K)}^{(e)} & =(4, X(X+2)) \\
\operatorname{Ann}_{W(K)}^{(o)} & =(4,(X-1)(X+1)) \\
\operatorname{Ann}_{W(K)} & =(4,(X-1) X(X+1)(X+2)) .
\end{aligned}
$$

(3) If $s(K)=4$, then

$$
\begin{aligned}
& \operatorname{Ann}_{W(K)}^{(e)}=(8,4 X, X(X+2)) \\
& \operatorname{Ann}_{W(K)}^{(o)}=(8,4(X+1),(X-1)(X+1)), \\
& \operatorname{Ann}_{W(K)}=(8,4 X(X+1),(X-1) X(X+1)(X+2)) .
\end{aligned}
$$


Proof. The cases $s(K)=1$ and $s(K)=2$ are clear. Assume that $s(K)=4$. We know that $X(X+2)$ annihilates every even-dimensional quadratic form over $K$. But if $\varphi$ is a form over $K$ such that $\{\varphi\}$ is annihilated by $2 X$, then $\{\varphi \perp 2\}$ is not annihilated by $2 X$. Now $2(X+2)$ annihilates $\{\varphi \perp 2\}$ but not $\{\varphi\}$. Hence $2(X+k) \notin \operatorname{Ann}_{W(K)}^{(e)}$ for all $k \in 2 \mathbb{Z}$. As $4 X \in \operatorname{Ann}_{W(K)}^{(e)}$ the claim follows for the even-dimensional case. The odd-dimensional case can be shown in an analogous fashion.

4.6 Remark. The results from the previous corollary coincide with the results presented by Ongenae and van Geel in [OG97, table 1]. There it is stated that

$$
\begin{aligned}
\operatorname{Ann}_{W(K)}^{(e)}= & \left(2, X^{2}\right) \\
& \text { if } s(K)=1 \text { and } I(K) \neq 0, \\
\operatorname{Ann}_{W(K)}^{(e)}= & \left(4, X(X+2), X^{3}\right)=(4, X(X+2)) \\
& \text { if } s(K)=2,2 I^{2}(K)=0 \text { and } 2 I(K) \neq 0, \\
\operatorname{Ann}_{W(K)}^{(e)}= & \left(8,4 X, X(X+2), X^{3}\right)=(8,4 X, X(X+2)) \\
& \text { if } s(K)=4,2 I^{3}(K)=0 \quad \text { and } \quad 2 I^{2}(K)=0 .
\end{aligned}
$$

In the case that $s(K)=1$ it is clear that the previous corollary represents a special case of the results in [OG97] since $I(K) \neq 0$ if $K$ is local.

Next we consider the case $s(K)=2$. Obviously we have $2 I^{2}(K)=0$ since $2 I^{2}(K) \subset I^{3}(K)=0$. Furthermore -1 is not a square in $K$. Let $\pi \in K$ be a uniformiser. Since $K$ is local it follows that $\langle 1,1, \pi, \pi\rangle=2 \otimes\langle 1, \pi\rangle$ is up to isometry the unique anisotropic 4-dimensional form over $K$ (compare for example [Lam05, chapter VI, theorem 2.2]). This implies that $2 I(K) \neq 0$.

Finally let $s(K)=4$. Then obviously $I^{3}(K)=0$. Furthermore we know that $I^{2}(K)$ consists of the two elements 0 and $\{\varphi\}$, where $\varphi$ is up to isometry the unique anisotropic 4-dimensional quadratic form over $K$. Since $s(K)=4$ we must have $4 \times\langle 1\rangle \cong \varphi$. This implies $2 \times \varphi \sim 0$ and hence $2 I^{2}(K)=0$.

\section{Global Fields}

In this section we will make use of the previous two sections to determine the annihilating ideal of a quadratic form $\varphi$ over a global field $K$ with the help of the Hasse-Minkowski Theorem ([Lam05, chapter VI, Hasse-Minkowski-Principle 3.1]).

Let $K$ be a global field, and let $V$ be the set of equivalence classes of absolute values of $K$. For every $\nu \in V$ choose a fixed representative $|\cdot|_{\nu}: K \rightarrow \mathbb{R}$ of the class $\nu$. Denote by $K_{\nu}$ the completion of $K$ with respect to $|\cdot|_{\nu}$. We can write $V$ as the disjoint union $V=V_{\mathbb{R}} \cup V_{\mathbb{C}} \cup V_{\text {fin }}$, where $K_{\nu}=\mathbb{R}$ for all $\nu \in V_{\mathbb{R}}, K_{\nu}=\mathbb{C}$ for all $\nu \in V_{\mathbb{C}}$, and $K_{\nu}$ is local with finite residue field for all $\nu \in V_{\text {fin }}$.

If $\varphi$ is a quadratic form over $K$ and $P \in \mathbb{Z}[X]$, then it follows from the Hasse-Minkowski Theorem that $P$ is an annihilating polynomial of $[\varphi]$, respectively $\{\varphi\}$, if and only if $P$ is an annihilating 
polynomial of $\left[\varphi_{K_{\nu}}\right]$, respectively $\left\{\varphi_{K_{\nu}}\right\}$, for all $\nu \in V$. This implies

$$
\operatorname{Ann}_{[\varphi]}=\bigcap_{\nu \in V} \operatorname{Ann}_{\left[\varphi_{K_{\nu}}\right]} \quad \text { and } \quad \operatorname{Ann}_{\{\varphi\}}=\bigcap_{\nu \in V} \operatorname{Ann}_{\left\{\varphi_{K_{\nu}}\right\}} .
$$

5.1 Example. We need to study annihilating polynomials of quadratic forms over $\mathbb{R}$ and $\mathbb{C}$.

(1) Let $\varphi$ be an $n$-dimensional quadratic form over $\mathbb{R}$. There exists only one signature homomorphism $W(\mathbb{R}) \rightarrow \mathbb{Z}$. Let $s \in \mathbb{Z}$ be the signature of $\varphi$. Since $\widehat{W}(\mathbb{R})$ and $W(\mathbb{R})$ are torsion free, it follows from example 3.8 that

$$
\operatorname{Ann}_{[\varphi]}= \begin{cases}(X-n) & \text { if } s=n \\ ((X-n)(X-s)) & \text { otherwise }\end{cases}
$$

and

$$
\operatorname{Ann}_{\{\varphi\}}=(X-s) .
$$

(2) Now let $\varphi$ be an $n$-dimensional quadratic form over $\mathbb{C}$. If $n$ is even, then $\varphi$ is hyperbolic. For $n$ odd we obtain $\varphi \sim\langle 1\rangle$. Hence

$$
\operatorname{Ann}_{[\varphi]}=(X-n)
$$

and

$$
\operatorname{Ann}_{\{\varphi\}}= \begin{cases}(2, X) & \text { for } n \text { even } \\ (2, X+1) & \text { for } n \text { odd }\end{cases}
$$

Now the signature homomorphisms $W(K) \rightarrow \mathbb{Z}$ are in one-to-one correspondence with the absolute values $\nu \in V_{\mathbb{R}}$. More specifically, for every ring homomorphism $\chi: W(K) \rightarrow \mathbb{Z}$ there exists a unique $\nu \in V_{\mathbb{R}}$ such that $\chi$ equals the concatenation $W(K) \rightarrow W\left(K_{\nu}\right) \rightarrow \mathbb{Z}$, where the first map is induced by the completion $K \hookrightarrow K_{\nu}$ and the second map is the unique signature homomorphism $W\left(K_{\nu}\right) \rightarrow \mathbb{Z}$. We denote the signature homomorphism corresponding to a $\nu \in V_{\mathbb{R}}$ by $\operatorname{sign}_{\nu}$. For a quadratic form $\varphi$ over $K$ this implies that $P \in \mathbb{Z}[X]$ is an annihilating polynomial of $\left[\varphi_{K_{\nu}}\right]$, respectively $\left\{\varphi_{K_{\nu}}\right\}$, for all $\nu \in V_{\mathbb{R}}$ if and only if $P \in\left(Q_{[\varphi]}\right)$, respectively $P \in\left(Q_{\{\varphi\}}\right)$. Hence, once we have calculated the embracing polynomial, we can neglect the real completions of $K$.

Example 5.1.(2) shows that $Q_{[\varphi]}$ annihilates $\left[\varphi_{K_{\nu}}\right]$ for all $\nu \in V_{\mathbb{C}}$. Furthermore it becomes clear by considering theorem 4.3 that $\operatorname{Ann}_{\left\{\varphi_{K_{\mu}}\right\}} \subset \operatorname{Ann}_{\left\{\varphi_{K_{\nu}}\right\}}$ for all $\mu \in V_{\text {fin }}$ and $\nu \in V_{\mathbb{C}}$, i.e. every polynomial in $\mathbb{Z}[X]$ that annihilates $\left\{\varphi_{K_{\mu}}\right\}$ also annihilates $\left\{\varphi_{K_{\nu}}\right\}$. These observations show that we do not need to consider the complex completions of $K$.

5.2 Proposition. Let $K$ be a global field, and let $\varphi$ be a quadratic form over $K$. We have

$$
\operatorname{Ann}_{[\varphi]}=\left(Q_{[\varphi]}\right) \cap \bigcap_{\nu \in V_{\text {fin }}} \operatorname{Ann}_{\left[\varphi_{K_{\nu}}\right]}
$$

and

$$
\operatorname{Ann}_{\{\varphi\}}=\left(Q_{\{\varphi\}}\right) \cap \bigcap_{\nu \in V_{\text {fin }}} \operatorname{Ann}_{\left\{\varphi_{K_{\nu}}\right\}} .
$$


By combining the previous proposition and theorem 4.2 we obtain:

5.3 Theorem. Let $K$ be a global field, and let $\varphi$ be an $n$-dimensional quadratic form over $K$.

(1) If $\varphi \cong n$, then $\operatorname{Ann}_{[\varphi]}=(X-n) \subset \mathbb{Z}[X]$.

(2) If $\varphi \neq n$, and

(a) if $\left|S_{[\varphi]}\right|=1$, then

$$
\operatorname{Ann}_{[\varphi]}= \begin{cases}\left(2(X-n),(X-n)^{2}\right) & \text { if } \operatorname{det}(\varphi) \text { is a sum of } \\ (4(X-n),(X-n)(X-n+2)) & \text { two squares in } K\end{cases}
$$

(b) if $\left|S_{[\varphi]}\right|=2$, then

$$
\operatorname{Ann}_{\varphi}= \begin{cases}\left(2(X-s)(X-n),(X-s)(X-n)^{2}\right) & \text { if } s \equiv n(\text { mod } 4) \text { and } \\ & \text { tet } \varphi) \text { is not a sum of } \\ ((X-s)(X-n)) & \text { otherwise, }\end{cases}
$$

where $S_{\varphi}=\{s, n\}$.

(c) if $\left|S_{[\varphi]}\right| \geq 3$, then $\operatorname{Ann}_{[\varphi]}=\left(Q_{[\varphi]}\right)$.

Proof. The points (1) and (2).(c) are clear.

Let $a \in K^{*}$. The Hasse-Minkowski Theorem states that $\langle 1,1,-a\rangle$ is isotropic if and only if $\langle 1,1,-a\rangle_{K_{\nu}}$ is isotropic for all $\nu \in V$. In other words $a$ is a sum of two squares in $K$ if and only if $a$ is a sum of two squares in $K_{\nu}$ for all $\nu \in V$. Obviously we do not have to consider any complex closures of $K$, and for the real closures we know that $a$ is a sum of two squares in $K_{\nu}$ for $\nu \in V_{\mathbb{R}}$ if and only if $a$ is positive with respect to $\nu$. Altogether this means that $a$ is a sum of two squares in $K$ if and only if $a$ is a sum of two squares in $K_{\mu}$ for all $\mu \in V_{\text {fin }}$ and $a$ is positive with respect to all $\nu \in V_{\mathbb{R}}$.

Let $\left|S_{[\varphi]}\right|=1$. This implies that $\operatorname{sign}_{\nu}(\varphi)=n$ for all $\nu \in V_{\mathbb{R}}$. In other words the entries of any diagonal representation of $\varphi$ are all positive with respect to all $\nu \in V_{\mathbb{R}}$. Hence $\operatorname{det}(\varphi)$ is positive with respect to all $\nu \in V_{\mathbb{R}}$, and $\operatorname{det}(\varphi)$ is a sum of two squares in $K$ if and only if $\operatorname{det}\left(\varphi_{\mu}\right)$ is a sum of two squares in $K_{\mu}$ for all $\mu \in V_{\text {fin }}$. Point (2).(a) now follows directly from theorem 4.2 and the Hasse-Minkowski Theorem.

Now consider the case $\left|S_{[\varphi]}\right|=2$. If either $s \equiv n+2(\bmod 4)$ or $s \equiv n(\bmod 4)$ and $\operatorname{det}(\varphi)$ is a sum of two squares in $K$ then our calculations concerning the Clifford invariant imply that $Q_{[\varphi]}=(X-s)(X-n)$ annihilates $\left[\varphi_{K_{\mu}}\right]$ for all $\mu \in V_{\text {fin }}$. Hence it follows from our previous observations that $\operatorname{Ann}_{[\varphi]}=((X-s)(X-n))$.

Finally we consider the case that $n \equiv s(\bmod 4)$ and $\operatorname{det}(\varphi)$ is not a sum of two squares in $K$. The fact that $n \equiv s(\bmod 4)$ implies that for all $\nu \in V_{\mathbb{R}}$ any diagonal representation of $\varphi$ has an even number of entries that are negative with respect to $\nu$. Therefore $\operatorname{det}(\varphi)$ is positive with respect to all $\nu \in V_{\mathbb{R}}$, and $\operatorname{det}(\varphi)$ is a sum of two squares in $K$ if and only if $\operatorname{det}\left(\varphi_{K_{\mu}}\right)$ is a sum 
of two squares in $K_{\mu}$ for all $\mu \in V_{\text {fin }}$. Since $\operatorname{det}(\varphi)$ is not a sum of two squares in $K$, there exists a $\mu_{0} \in V_{\text {fin }}$ such that $\operatorname{det}\left(\varphi_{K_{\mu_{0}}}\right)$ is not a sum of two squares in $K_{\mu_{0}}$. Our calculations concerning the Clifford invariant imply that $Q_{[\varphi]}=(X-s)(X-n)$ does not annihilate $\left[\varphi_{K_{\mu_{0}}}\right]$. Hence $Q_{[\varphi]}$ does not annihilate $[\varphi]$. Since $2 Q_{[\varphi]}$ and $(X-n) Q_{[\varphi]}$ do annihilate $\left[\varphi_{K_{\mu}}\right]$ for all $\mu \in V_{\text {fin }}$, it is now clear that those two polynomials generate $\operatorname{Ann}_{[\varphi]}$.

Again an analogous theorem for $\mathrm{Ann}_{\{\varphi\}}$ demands the distinction of even more cases.

5.4 Theorem. Let $K$ be a global field, and let $\varphi$ be an $n$-dimensional quadratic form over $K$.

(1) If $\varphi \sim r$ for some $r \in \mathbb{Z}$, then

$$
\operatorname{Ann}_{\{\varphi\}}= \begin{cases}(X-r) & \text { if } s(K)=\infty \\ (2 s(K), X-r) & \text { otherwise. }\end{cases}
$$

(2) If $\varphi \nsim r$ for all $r \in \mathbb{Z}$,

(a) if $\left|S_{\{\varphi\}}\right|=0$, and

(i) if $s(K)=1$, then

$$
\operatorname{Ann}_{\{\varphi\}}= \begin{cases}\left(2, X^{2}\right) & \text { if } n \text { is even } \\ \left(2,(X+1)^{2}\right) & \text { if } n \text { is odd. }\end{cases}
$$

(ii) if $s(K)=2$, then for $n$ even

$$
\operatorname{Ann}_{\{\varphi\}}= \begin{cases}\left(4,2 X, X^{2}\right) & \text { if } d(\varphi) \text { is a sum of } \\ (4, X(X+2)) & \text { two squares in } K\end{cases}
$$

and for $n$ odd

$$
\operatorname{Ann}_{\{\varphi\}}= \begin{cases}\left(4,2(X+1),(X+1)^{2}\right) & \text { if } d(\varphi) \text { is a sum of } \\ (4,(X-1)(X+1)) & \text { otherwise. }\end{cases}
$$

(iii) if $s(K)=4$, then for $n$ even

$$
\operatorname{Ann}_{\{\varphi\}}= \begin{cases}\left(8,2 X, X^{2}\right) & \text { if } d(\varphi) \text { is a sum of } \\ & \text { two squares in } K, \\ \left(8,2(X+2),(X+2)^{2}\right) & \text { if }-d(\varphi) \text { is a sum of } \\ & \text { two squares in } K, \\ (8,4 X, X(X+2)) & \text { otherwise, }\end{cases}
$$

and for $n$ odd

$$
\operatorname{Ann}_{\{\varphi\}}= \begin{cases}\left(8,2(X-1),(X-1)^{2}\right) & \text { if } d(\varphi) \text { is a sum of } \\ & \text { two squares in } K, \\ \left(8,2(X+1),(X+1)^{2}\right) & \text { if }-d(\varphi) \text { is a sum of } \\ (8,4(X+1),(X-1)(X+1)) & \text { two squares in } K, \\ \text { otherwise, }\end{cases}
$$


(b) if $\left|S_{\{\varphi\}}\right|=1$, then

$$
\operatorname{Ann}_{\{\varphi\}}= \begin{cases}\left(2(X-r),(X-r)^{2}\right) & \text { if }(-1)^{\frac{r(r-1)}{2} d(\varphi) \text { is a sum }} \\ (4(X-r),(X-r)(X-r+2)) & \text { of two squares in } K, \\ \text { otherwise, }\end{cases}
$$

where $S_{\{\varphi\}}=\{r\}$.

(c) if $\left|S_{\{\varphi\}}\right|=2$, then

$$
\operatorname{Ann}_{\{\varphi\}}= \begin{cases}(2(X-s)(X-r), & \text { if } r \equiv s(\bmod 4) \text { and } \\ \left.(X-s)(X-r)^{2}\right) & (-1)^{\frac{r(r-1)}{2}} d(\varphi) \text { is not a } \\ ((X-s)(X-r)) & \text { sum of two squares in } K,\end{cases}
$$

where $S_{\{\varphi\}}=\{r, s\}$.

(d) if $\left|S_{\{\varphi\}}\right| \geq 3$, then $\operatorname{Ann}_{\{\varphi\}}=\left(Q_{\{\varphi\}}\right)$.

Proof. The points (1) and (2).(d) are clear. Point (2).(a) follows directly from proposition 5.2, theorem 4.3, and the Hasse-Minkowski Theorem since in this case $V_{\mathbb{R}}=\varnothing$. For point (2).(a).(iii) we have to take into account the possibility that neither $d(\varphi)$ nor $-d(\varphi)$ is a sum of 2 squares in $K$. In this case we obtain the generators by the same reasoning that we employed for the case $s(K)=4$ in the proof of corollary 4.5 .

Now assume that $S_{\{\varphi\}}=\{r\}$. There exist $a, b \in \mathbb{N}_{0}$ such that $n=a+b$ and $r=a-b$. The determinant $\operatorname{det}(\varphi)$ is negative with respect to all $\nu \in V_{\mathbb{R}}$ if and only if $b$ is odd. Simple calculations show that

$$
(-1)^{\frac{r(r-1)}{2}} d(\varphi)=(-1)^{a^{2}-a+b^{2}} \operatorname{det}(\varphi)=(-1)^{b} \operatorname{det}(\varphi) .
$$

This implies that $(-1)^{\frac{r(r-1)}{2}} d(\varphi)$ is positive with respect to all $\nu \in V_{\mathbb{R}}$. Hence $(-1)^{\frac{r(r-1)}{2}} d(\varphi)$ is a sum of two squares in $K$ if and only if it is a sum of two squares in $K_{\mu}$ for all $\mu \in V_{\text {fin }}$. Point (2).(b) now follows from example 4.1.(2) and the Hasse-Minkowski Theorem.

Next consider the case $S_{\{\varphi\}}=\{s, r\}$ with $s \neq r$. If $s \equiv r+2(\bmod 4)$, then it follows from our calculations that $(X-s)(X-r)$ annihilates $\left\{\varphi_{K_{\mu}}\right\}$ for all $\mu \in V_{\text {fin }}$. Since $(X-s)(X-r)=Q_{\{\varphi\}}$ we have $\operatorname{Ann}_{\{\varphi\}}=((X-s)(X-r))$. If $r \equiv s(\bmod 4)$ and $(-1)^{\frac{r(r-1)}{2}} d(\varphi)$ is a sum of two squares in $K$ then the same holds over $K_{\mu}$ for all $\mu \in V_{\text {fin }}$. Thus it follows from 4.1.(2) that $(X-s)(X-r)$ annihilates $\{\varphi\}$. Hence we have again $\operatorname{Ann}_{\{\varphi\}}=((X-s)(X-r))$.

Finally consider the case that $s \equiv r(\bmod 4)$ and $(-1)^{\frac{r(r-1)}{2}} d(\varphi)$ is not a sum of two squares in $K$. By our observation earlier in this proof we know that $(-1)^{\frac{r(r-1)}{2}} d(\varphi)$ is positive for all $\nu \in V_{\mathbb{R}}$ with $\operatorname{sign}_{\nu}(\{\varphi\})=r$. Since $s \equiv r(\bmod 4)$ the same holds for all $\nu \in V_{\mathbb{R}}$ with $\operatorname{sign}_{\nu}(\{\varphi\})=s$. Thus there must exist a $\mu_{0} \in V_{\text {fin }}$ such that $(-1)^{\frac{r(r-1)}{2}} d\left(\varphi_{K_{\mu_{0}}}\right)$ is not a sum of two squares in $K_{\mu_{0}}$. By our calculations and the Hasse-Minkowski Theorem $Q_{\{\varphi\}}=(X-s)(X-r)$ does not annihilate $\{\varphi\}$. This implies that we must have $\operatorname{Ann}_{\{\varphi\}}=\left(2 Q_{\{\varphi\}},(X-r) Q_{\{\varphi\}}\right)$, which completes the proof of point (2).(c). 
5.5 Remark. We can now use the last theorem to formulate a result about $\operatorname{Ann}_{W(K)}^{(e)}, \operatorname{Ann}_{W(K)}^{(o)}$, and $\mathrm{Ann}_{W(K)}$ in the case that $K$ is global with $s(K)<\infty$. But since we will obtain, depending on $s(K)$, exactly the same ideals as in the local case, we simply refer to corollary 4.5 .

\section{References}

[BP97] Beaulieu, Patricia W. ; Palfrey, Thomas C.: The Galois number. In: Math. Ann. 309 (1997), Nr. 1, pp. 81-96

[Con87] Conner, Pierre E.: A proof of the conjecture concerning algebraic Witt classes. 1987. - Preprint

[Hur89] HurRelbrink, Jürgen: Annihilating polynomials for group rings and Witt rings. In: Canad. Math. Bull. 32 (1989), Nr. 4, pp. 412-416

[Kne77] Knebusch, Manfred: Generic Splitting of Quadratic Forms II. In: Proc. London Math. Soc. 34 (1977), pp. 1-31

[KrW72] Knebusch, Manfred ; Rosenberg, Alex ; Ware, Roger: Structure of Witt rings and quotients of Abelian group rings. In: Amer. J. Math. 94 (1972), Nr. 1, pp. 119-155

[Lam05] LAm, Tsit-Yuen: Grad. Stud. Math.. Vol. 67: Introduction to Quadratic Forms over Fields. Amer. Math. Soc., 2005

[Lew87] LewIS, David W.: Witt rings as integral rings. In: Invent. Math. 90 (1987), pp. 631-633

[Lew92] Lewis, David W.: Annihilating Polynomials and Positive Forms. In: Canad. Math. Bull. 35 (1992), Nr. 1, pp. 103-107

[Lew01] LewIs, David W.: Annihilating Polynomials for Quadratic Forms. In: Int. J. Math. Math. Sci. 27 (2001), Nr. 7, pp. 449-455

[LM00] Lewis, David W. ; MCGarraghy, Seán: Annihilating polynomials, étale algebras, trace forms and the Galois number. In: Arch. Math. 75 (2000), pp. 116-120

[OG97] Ongenae, Veerle; van Geel, Jan: Polynomials annihilating the Witt ring. In: Math. Nachr. 185 (1997), pp. 213-226

[Pfi95] Pfister, Albrecht: London Math. Soc. Lecture Note Ser.. Vol. 217: Quadratic Forms with Applications to Algebraic Geometry and Topology. Cambridge Univ. Press, 1995

[Réd67] RÉDeI, László: Algebra, Volume 1. Pergamon Press, 1967

[Rüh08] RüHL, Klaas-Tido: Annihilating Polynomials of Excellent Quadratic Forms. In: Arch. Math. 90 (2008), Nr. 3, pp. 217-222

[Sch85] Scharlau, Winfried: Grundlehren Math. Wiss.. Vol. 270: Quadratic and Hermitian Forms. Springer, 1985 
[Sze52] Szekeres, G.: A canonical basis for the ideals of a polynomial domain. In: Amer. Math. Monthly 6 (1952), pp. 379-386

[Wan07] DE Wannemacker, Stefan: Annihilating polynomials for quadratic forms and Stirling numbers of the second kind. In: Math. Nachr. 280 (2007), pp. 1257-1267 